\title{
Intensive care in thoracic oncology
}

\author{
Anne-Pascale Meert ${ }^{1}$, Bogdan Grigoriu ${ }^{1}$, Marc Licker ${ }^{2}$, Paul E. Van Schil ${ }^{3}$ and \\ Thierry Berghmans ${ }^{1}$
}

Number 6 in the Series "Multidisciplinary questions in thoracic oncology: the team experience"

Edited by J-P. Sculier

\begin{abstract}
Affiliations: 'Service des Soins Intensifs et Urgences Oncologiques et Oncologie Thoracique, Institut Jules Bordet, Université Libre de Bruxelles (ULB), Brussels, Belgium. ${ }^{2}$ Dept of Anesthesiology, Pharmacology and Intensive Care, University Hospitals of Geneva, Geneva, Switzerland. ${ }^{3}$ Dept of Thoracic and Vascular Surgery, Antwerp University Hospital, Edegem (Antwerp), Antwerp, Belgium.
\end{abstract}

Correspondence: Anne-Pascale Meert, Institut Jules Bordet, 1 rue Héger-Bordet, 1000 Bruxelles, Belgium. E-mail: ap.meertlabordet.be

@ERSpublications

ICU should be proposed to lung cancer patients after discussion between the patient and the multidisciplinary team http://ow.ly/TWZ3308SB4g

Cite this article as: Meert A-P, Grigoriu B, Licker M, et al. Intensive care in thoracic oncology. Eur Respir J 2017; 49: 1602189 [https://doi.org/10.1183/13993003.02189-2016].

ABSTRACT The admission of lung cancer patients to intensive care is related to postprocedural/ postoperative care and medical complications due to cancer or its treatment, but is also related to acute organ failure not directly related to cancer.

Despite careful preoperative risk management and the use of modern surgical and anaesthetic techniques, thoracic surgery remains associated with high morbidity, related to the extent of resection and specific comorbidities. Fast-tracking processes with timely recognition and treatment of complications favourably influence patient outcome. Postoperative preventive and therapeutic management has to be carefully planned in order to reduce postoperative morbidity and mortality.

For patients with severe complications, intensive care unit (ICU) mortality rate ranges from $13 \%$ to $47 \%$, and hospital mortality ranges from $24 \%$ to $65 \%$. Common predictors of in-hospital mortality are severity scores, number of failing organs, general condition, respiratory distress and the need for mechanical ventilation or vasopressors. When considering long-term survival after discharge, cancerrelated parameters retain their prognostic value.

Thoracic surgeons, anesthesiologists, pneumologists, intensivists and oncologists need to develop close and confident partnerships aimed at implementing evidence-based patient care, securing clinical pathways for patient management while promoting education, research and innovation. The final decision on admitting a patient with lung to the ICU should be taken in close partnership between this medical team and the patient and his or her relatives.

Received: Nov 092016 | Accepted after revision: Feb 062017

Previous articles in this series: No. 1: Malhotra J, Malvezzi M, Negri E, et al. Risk factors for lung cancer worldwide. Eur Respir J 2016; 48: 889-902. No. 2: McDonald F, De Waele M, Hendriks LEL, et al. Management of stage I and II nonsmall cell lung cancer. Eur Respir J 2017; 49: 1600764. No. 3: Leduc C, Antoni D, Charloux A, et al. Comorbidities in the management of patients with lung cancer. Eur Respir J 2017; 49: 1601721. No. 4: Zugazagoitia J, Molina-Pinelo S, Lopez-Rios F, et al. Biological therapies in nonsmall cell lung cancer. Eur Respir J 2017; 49: 1601520. No. 5: Calvayrac O, Pradines A, Pons E, et al. Molecular biomarkers for lung adenocarcinoma. Eur Respir J 2017; 49: 1601734.

Conflict of interest: None declared.

Copyright @ERS 2017 


\section{Introduction}

In Western countries, lung cancer is the primary cause of death by cancer [1], and $8-15 \%$ of cancer patients admitted to intensive care units (ICU) have lung cancer [2, 3]. Admission is related to postprocedural/postoperative care, medical complications due to cancer or its treatment and organ failure not related to cancer. The prognosis of ICU cancer patients is determined by acute physiological disturbances leading to intensive care, but not the underlying neoplastic condition [4-6], while survival after recovery is related to the underlying neoplastic disease.

Changes in the management of lung cancer patients, health costs and budgetary constraints induce substantial modifications to ICU usage. Traditionally, surgical lung cancer patients were routinely admitted to the ICU for the immediate postoperative period, but currently we are facing the development of high-dependency (HDU) and post-anaesthesia care units (PACUs). Admitting advanced lung cancer patients to the ICU remains a challenging issue, for which new therapeutic developments with very active targeted therapies have to be taken into account.

This narrative review repositions the current role of the ICU in managing patients with lung cancer, focusing on three major end-points: selection of patients at risk after major surgical procedures; prognosis of lung cancer patients admitted to the ICU; and specific complications leading to ICU admission.

\section{Selection of patients at risk after surgical procedures for ICU admission}

Postoperative mortality rates after major thoracic surgical procedures range between $2 \%$ and $5 \%$, and cardiopulmonary morbidity between $20 \%$ and $40 \%$, resulting in prolonged hospital stays and increased healthcare costs [7].

Post-operative admission to the ICU, HDU or PACU is based on the clinician's judgment for prediction of "avoidable" major complications modulated by available resources. Combining patient- and procedure-related risks approximates the overall risk profile (figure 1).

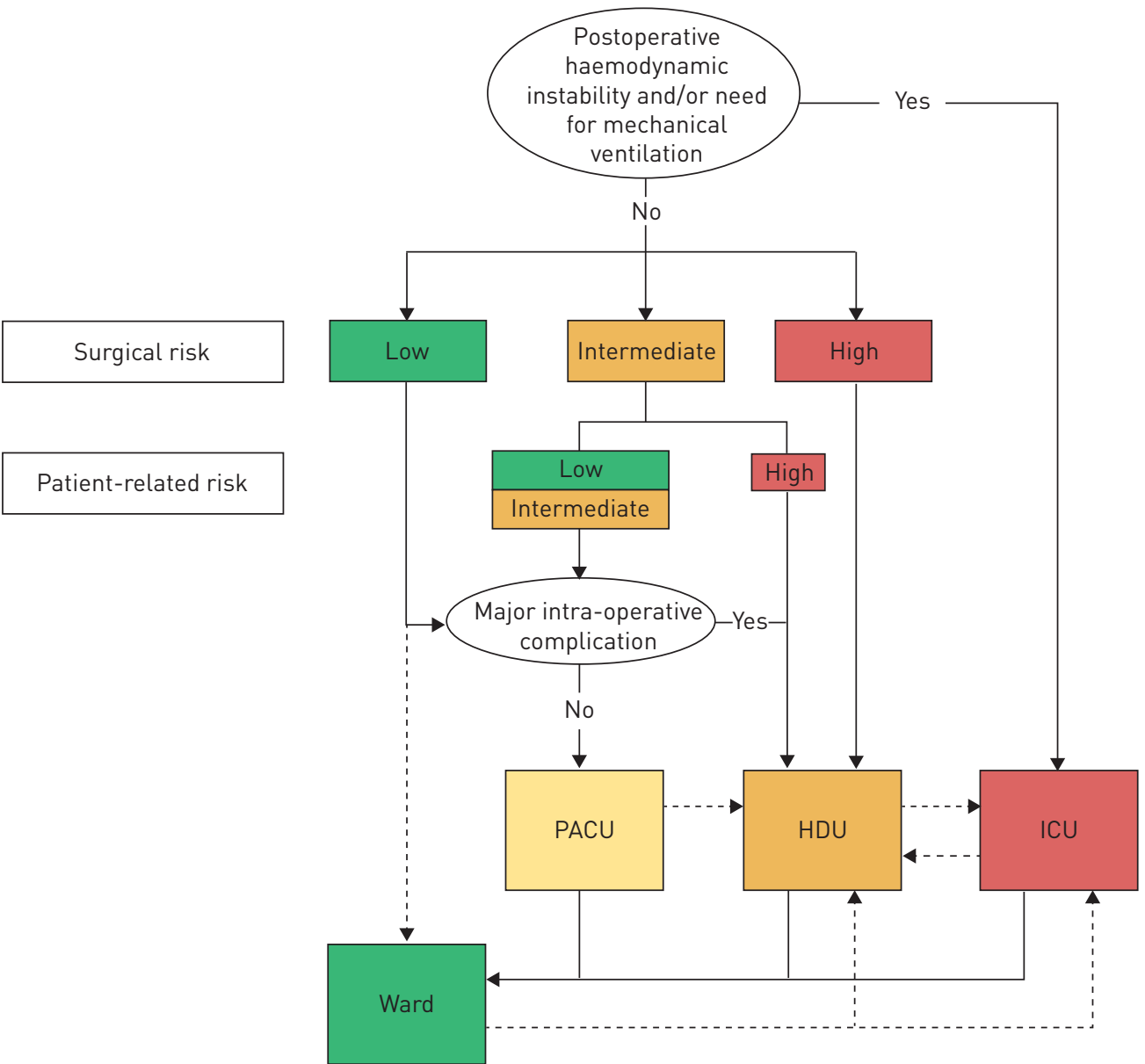

FIGURE 1 Post-thoracotomy patient triage. PACU: post-anaesthesia care unit; HDU: high-dependency unit; ICU: intensive care unit. Reproduced and modified from [7]. 


\section{TABLE 1 Summary of risk factors indicating a high risk of postoperative complications}

\section{Patient-related risk factors}

ASA physical status $\geqslant 3$

S-MPM $\geqslant 6$ points

$\mathrm{RCRI} \geqslant 2$ points

ThRCRI $>1.5$ points

ARISCAT $>45$ points

Preoperative FEV $1<60 \%$

ppo-FEV $1<30 \%$

ppo- $D$ LCO $<30 \%$

Peak $V^{\prime} \mathrm{O}_{2}<12 \mathrm{~mL} \cdot \mathrm{kg}^{-1} \cdot \mathrm{min}^{-1}$

Liver dysfunction?

\section{Procedure-related risk factors}

High-risk procedure

Major intraoperative complication"

Low level of operator and hospital expertise

Emergency operation

ASA: American Society of Anesthesiologists; S-MPM: surgical mortality probability model; RCRI: revised cardiac risk index; ThRCRI: thoracic RCRI; ARISCAT: Assess Respiratory Risk in Surgical Patients in Catalonia risk index; $F E V$ 1: forced expiratory volume in $1 \mathrm{~s}$; ppo: predictive postoperative; DLco: diffusing capacity of the lung for carbon monoxide; $V^{\prime} \mathrm{O}_{2}$ : oxygen uptake. \#: refractory hypotension and/or hypoxaemia, myocardial ischaemia, cardiac arrhythmias requiring treatment, major haemorrhage or bronchial aspiration; ${ }^{\text {I: }}$ according to [8-10].

Risk factors for surgical complications and risk stratification

Risk factors are summarised in tables 1, 2 and 3.

Patient-related risk factors

General risk scores

The American Society of Anesthesiologists classification of physical health (ASA) is universally applied for assessing the risk of post-operative morbidity and mortality of patients requiring any surgical, therapeutic or diagnostic procedure. However, high risk (ASA >II) is associated with large interobserver variability and poor specificity, which preclude accurate estimation for individual patients [11].

TABLE 2 Summary of risk scores

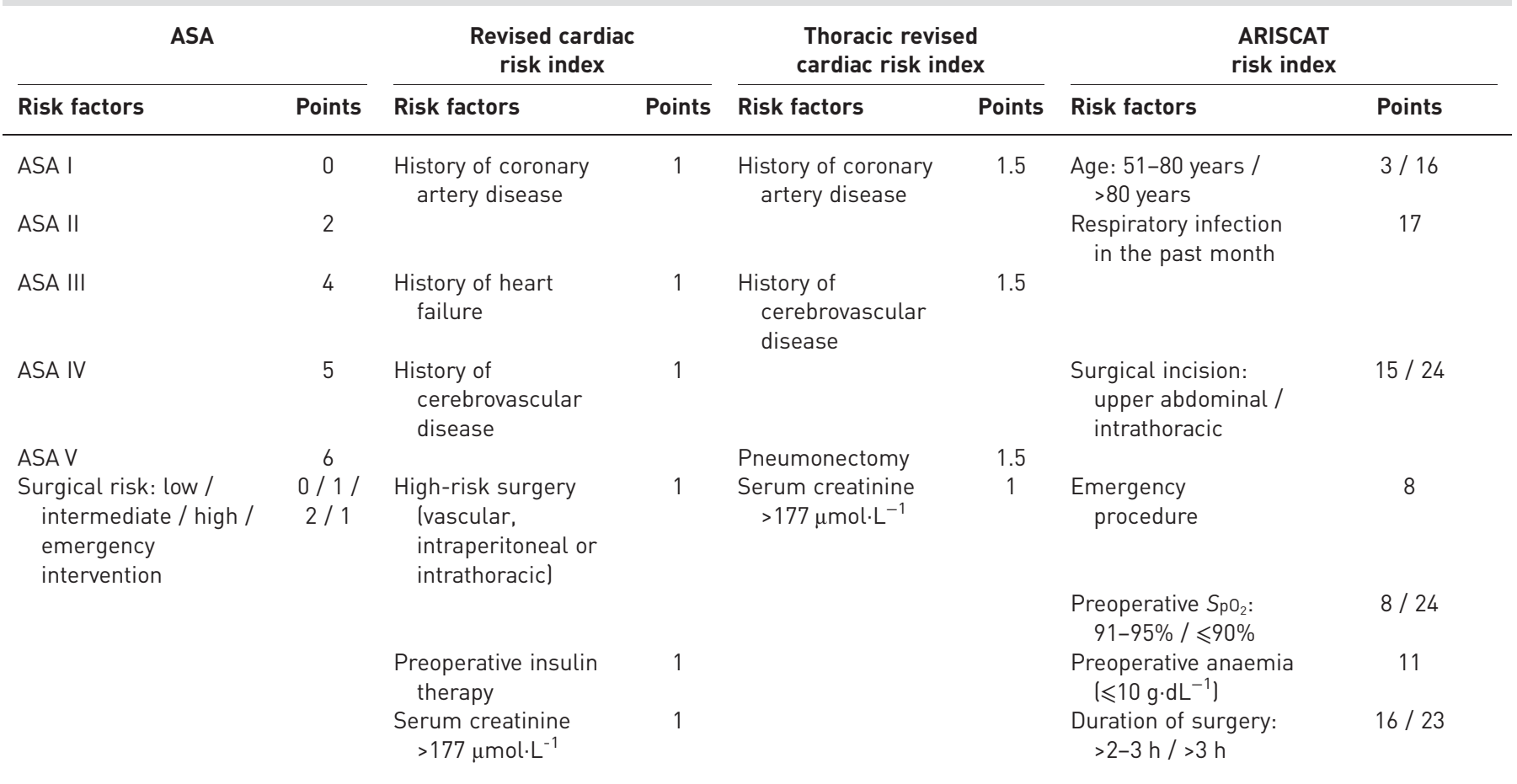

ASA: American Society of Anesthesiologists' classification of physical health; ARISCAT: Assess Respiratory Risk in Surgical Patients in Catalonia; $\mathrm{SpO}_{2}$ : arterial oxygen saturation measured by pulse oximetry. 
The Charlson comorbidity index (CCI), composed of 19 weighted medical diagnoses, is a valid predictor of 1 -year mortality in medical patients; a score $>5$ being associated with 1 -year mortality $>50 \%$ [12]. Among lung cancer patients undergoing curative resection, a CCI score $\geqslant 3$ was associated with a 10 -fold greater incidence of major complications [13]. The surgical mortality probability model [14] is used to predict all-cause post-operative mortality at 30 days [15] (tables 2 and 3).

Three further scores, more specifically designed for thoracic surgery, have to be considered. The cardiopulmonary risk index is a combination of cardiac and pulmonary risk factors [16]. The EVAD score utilises pulmonary function test data (forced expiratory volume in $1 \mathrm{~s}\left(\mathrm{FEV}_{1}\right.$ ) and diffusing capacity of the lung for carbon monoxide (DLCO)) and patient age to predict the likelihood of complications after major lung resection [17]. The "thoracoscore", derived from the French national thoracic surgery database EPITHOR incorporates nine independent risk factors to predict in-hospital mortality [18].

\section{Cardiovascular risk scores}

The revised cardiac risk index (RCRI) was developed for prediction of major cardiac complications in nonemergency noncardiac surgery [19] (tables 2 and 3). A RCRI index $\geqslant 3$ is associated with major postoperative cardiac complications $(+11 \%)$. The thoracic RCRI for lung resections was derived from the original RCRI [20] (tables 2 and 3). The predictive power of both scores in patients undergoing lung resections is controversial. More recently, the myocardial infarction and cardiac arrest risk calculator [21] has identified five predictors of perioperative risk at 30 days and provides a more accurate cardiac risk prediction than RCRI, although no data for thoracic surgery are available.

\section{Pulmonary risk scores}

Postoperative pulmonary complications (PPCs) are a major cause of morbidity and mortality, possibly accounting for more mortality than cardiovascular complications.

The Assess Respiratory Risk in Surgical Patients in Catalonia (ARISCAT) study established a risk score for the development of PPCs in a cohort of surgical patients based on seven independent risk factors [22] (tables 2 and 3). The score was prospectively and externally validated across many European countries, with a satisfactory predictive power, especially for western European countries [23].

\section{Lung function tests}

Dyspnoea is correlated with the risk of postoperative mortality [24]. Standardised symptom-limited climbing of three flights of stairs without interruption is a simple cost-effective test to objectively determine

\begin{tabular}{ccc} 
TABLE 3 Interpretation of risk scores & \\
& Score & Mortality \\
& & \\
\hline \multirow{2}{*}{ ASA } & 1 & 0.01 \\
& 2 & 0.02 \\
& 3 & 0.07 \\
& 4 & 0.2 \\
& 5 & 1.5 \\
& 6 & 4 \\
& 7 & 10 \\
& 8 & 25 \\
9 & 50
\end{tabular}

Revised cardiac risk index

Thoracic revised cardiac risk index

ARISCAT risk index
0.01
0.02
0.2
1.5
4
10
50

$\begin{array}{cc}0 & 0.4(0.05-1.5) \\ 1 & 0.9(0.3-2.1) \\ 2 & 6.6(3.9-10.3) \\ \geqslant 3 & \geqslant 11(5.8-18.4) \\ 0 & 0.9 \\ 1-1.5 & 4.2 \\ 2-2.5 & 8 \\ >2.5 & 18\end{array}$

$<26 \quad$ Low 1.6 (0.6-2.6)

26-45 Intermediate $13.3(7.6-19)$

$>45 \quad$ High 42.1 (29.3-54.9)

Data are presented as \% or \% (95\% CI). ASA: American Society of Anesthesiologists classification of physical health; ARISCAT: Assess Respiratory Risk in Surgical Patients in Catalonia. 
cardiorespiratory reserve and may have superior predictive ability than traditional spirometry values [7]. Failure to perform this test warrants further lung function testing, while patients able to climb $\geqslant 22 \mathrm{~m}$ (five to six flights of stairs) have a low risk of postoperative complications, regardless of lung function test results [25].

A preoperative $\mathrm{FEV}_{1}<70 \%$ and a predictive postoperative (ppo) DLCO are reliable predictors of perioperative complications in thoracic surgery [26]. European Respiratory Society/European Society of Thoracic Surgery guidelines use ppo-FEV1 $<30 \%$ and ppo-DLCO $<30 \%$ to discriminates between normal and high-risk groups [7]. However, the calculated ppo-FEV1 may overestimate the FEV1 on the first postoperative day by $\sim 30 \%$ [27] and patients with a moderate to severe obstructive pulmonary syndrome may improve their respiratory dynamics after lung resection.

Peak oxygen uptake during a standardised effort allows the refinement of perioperative risk prediction, with values $>20 \mathrm{~mL} \cdot \mathrm{kg}^{-1} \cdot \mathrm{min}^{-1}$ allowing resection up to pneumonectomy, whereas values $<10 \mathrm{~mL} \cdot \mathrm{kg}^{-1} \cdot \mathrm{min}^{-1}$ predict a high risk of any type of lung resection [28] with a mortality rate that may exceed 20\% [29].

Age and frailty

Decline in organ function and physiological reserve with ageing is a major risk factor for perioperative morbidity and mortality. Sarcopenia affects muscles of the limbs, respiratory muscles and those controlling the upper airways. Obstructive sleep apnoea and occult aspiration occur more frequently in the context of underlying neurological disorders (e.g. previous stroke, dementia or Parkinson's disease) [30]. The risk of postoperative hypoxia and hypercapnia is increased because of altered chemosensitivity, respiratory muscle weakness and increased pulmonary shunting. Impaired thermogenesis favours the occurrence of wound infection, bleeding and cardiac ischaemia, resulting in prolonged postoperative recovery [27]. The risk of postoperative cognitive disorder is increased, especially with benzodiazepine premedication [31].

Frailty is a composite measure of geriatric conditions that may be a valuable aid in determining operability and planning of postoperative care. A multidimensional frailty score including nine items adapted from comprehensive geriatric assessment with a maximal value of 15 has been elaborated for predicting 1-year postoperative mortality [32]. A cut-off score of five allows practitioners to distinguish between high(mortality $>10 \%$ ) and low-risk populations. Although superior to the ASA score for prediction of 1-year mortality, its computation is time-consuming, and must be performed by a consultant familiar with the score.

\section{Procedure-related risk factors}

Lung resections

Thoracic surgery risk assessment focuses primarily on lung resections, particularly in the context of cancer surgery. Broadly, the more extensive the lung resection, the greater the risk of developing postoperative complications (table 4).

The greatest risk is associated with extended pneumonectomy (table 5). Overall, right-sided lung resection carries a higher risk of complications than a left-sided resection, owing to a greater propensity for bronchopleural fistula formation, a greater increase in right ventricular afterload and potential alterations in cardiac sympathicovagal balance $[33,34]$.

\section{Other thoracic surgical interventions}

Interventions requiring single or one-lung ventilation and a thoracotomy expose patients to cardiovascular complications, atelectasis, pneumonia and ventilator-induced lung injuries, leading to acute respiratory distress syndrome (ARDS).

\section{TABLE 4 Risk classification according to type of thoracic surgical procedure}

Low risk Intermediate risk High risk

Pleural drainage

Pleurodesis

Mediastinoscopy

Lung biopsy
Bullectomy

Pleural resection

Lobectomy

Segmentectomy

Wedge resection
Pneumonectomy

Extended lung resection

Trachea, bronchial resection

Mediastinal resections ${ }^{\#}$

Pneumonectomy

Pneumonectomy

Diaphragmatic resection

Lung volume reduction surgery

Lung transplantation

\footnotetext{
\#: oesophagectomy, mediastinal tumour resection and thymus resection.
} 
TABLE 5 Risk factors according to extent of lung resection

Source [ref.] Patients $\mathrm{n}$

1267 pneumonectomies

STS GTSD [33]

French National Database for Thoracic Surgery (EPITHOR) [34]

STS GTSD [35]

American National Cancer Database [36]
4498 pneumonectomies

18800 lung cancer

resections

120000 patients
Mortality

Risk factors
$5.6 \%$

$7.8 \%$

$2.2 \%$

Overall 30 -day mortality $3.4 \%$

for NSCLC resections;

$8.5 \%$ for pneumonectomies;

$4 \%$ for extended lobectomies and bilobectomies;

$2.6 \%$ for lobectomies;

$4.2 \%$ for wedge resections

STS: Society for Thoracic Surgeons; GTSD: General Thoracic Surgery Database; FEV1: forced expiratory volume in 1 s; ASA: American Society of Anesthesiologists' classification of physical health; NSCLC: nonsmall cell lung cancer.

A nomogram was validated to predict the occurrence and severity of postoperative complications after oesophagectomy [35] and proved useful for risk prediction in high-volume hospitals [37]. Independent risk factors are age, history of cerebrovascular or transient ischaemic attack, history of myocardial infarction, reduced FEV1, ECG changes and extensive surgery.

Lung or pleural biopsies and simple bullectomy with or without pleurodesis using video-assisted thoracic surgery (VATS) require short-term admission to a PACU for monitoring recovery from anaesthesia, lung re-expansion, titration of analgesics and detection of residual air leakage and atelectasis. Mediastinoscopies can generally be monitored in the PACU, with special attention to the risk of occult postoperative haemorrhage.

Additional surgical risk factors

There is little evidence to support the use of a muscle-sparing thoracotomy as opposed to a posterolateral thoracotomy, but incision length may be proportionally related to post-thoracotomy complications [38]. Given limited tissue trauma and consequent reduced neuroendocrine and inflammatory responses, VATS is associated with lower rates of perioperative mortality, morbidity (e.g. pneumonia and atrial arrhythmia) and length of stay [34]. In the absence of other major risk factors for postoperative complications, patients with a VATS lung resection are commonly managed in PACUs for monitoring and recovery from anaesthesia.

Operative mortality may be lower if board-certified thoracic surgeons have a minimal case load of procedures [39]. Differences in postoperative mortality rates between hospitals may also be explained by variation in the quality of postoperative patient management [40].

Surgery performed on an emergency basis has repeatedly been associated with worse postoperative outcomes. Various pre- and postoperative scores integrate this factor into risk stratification.

Finally, the occurrence of major intraoperative complications may require a higher level of postoperative monitoring and treatment than initially planned. Myocardial ischaemia, haemodynamically significant arrhythmias, refractory hypotension or hypoxaemia, bronchial aspiration and major bleeding are considered major complications that justify admission to a HDU or ICU.

\section{Anaesthetic management}

Improving patient outcome can be achieved by implementing perioperative risk minimisation strategies [8-10]: 1) titration of anaesthetic agents, based on monitoring brain activity; 2) adoption of lung-protective ventilatory settings; 3) control of haemodynamics and achievement of optimal oxygen transport to match metabolic demands (cerebral oxygen saturation by near-infrared spectroscopy); 4) control of normothermia and haemostasis; and 5) efficient pain control. 
The type and quality of postoperative pain control influences postoperative triage, since it influences the risk of postoperative cardiopulmonary complications and length of stay [41]. Thoracic epidural analgesia is considered the gold standard for pain management after open thoracic surgery as it has been associated with a lower incidence of postoperative pneumonia and a shorter duration of mechanical ventilation compared to systemic analgesia [42]. Thoracic paravertebral block, performed percutaneously by the anaesthetist or directly by the surgeon, may provide similar analgesia, at lower risk of hypotension and urinary retention [43]. In some institutions, refractory hypotension caused by the thoracic epidural treatment mandates transfer to the HDU or ICU. Over recent decades, the incidence of post-procedural complications has markedly decreased due to the additional benefit of epidural over systemic analgesia [42].

\section{Postoperative selection of patients for ICU, HDU or PACU admission}

For most patients, postoperative triage can be planned by taking into account preoperative patient- and procedure-related risk factors while the occurrence of intraoperative complications may modify the initial assessment.

\section{Scores and guidelines}

The surgical Apgar score (SAS) includes three intraoperative parameters: blood loss, lowest mean arterial pressure and lowest heart rate, shown to be useful in predicting postoperative major complications or 30-day mortality following noncardiac surgery [44]. So far, thoracic surgical patients have not been included in this exploratory population sample. The SAS has been validated successfully in single centres and in an international study across eight hospitals in eight countries [45]. A score $\geqslant 6$ may signal a high risk of postoperative complications and prompt the clinician to transfer a patient to the HDU or ICU.

The physiological and operative severity score for the enumeration of mortality and morbidity (POSSUM) was developed for audit purposes. It is based on 12 physiological and six operative parameters and allows prediction of in-hospital mortality and morbidity. It proved useful for prediction of postoperative complications in lung-resected patients [46]. Subsequently, the POSSUM score was improved and named Portsmouth-POSSUM (P-POSSUM) [47].

The American College of Critical Care Medicine issued guidelines regarding selection criteria of patients admitted to HDUs [48] and ICUs [49]. Admission to the HDU should be considered for "patients who, after major surgery, are hemodynamically stable, but may require fluid resuscitation and transfusion due to major fluid shifts" and "who require close nurse monitoring during the first $24 \mathrm{~h}$ ". Admission to the ICU is restricted for a minority of patients requiring "hemodynamic monitoring/ventilatory support or extensive nursing care" (figure 1). Changing the clinical pathway of thoracic surgical patients from the ICU to the PACU and HDU has been shown to be safe and cost-effective [50].

\section{Local characteristics}

The spectrum of postoperative units extends from day care and surgical wards to PACUs, HDUs and ICUs. Every hospital has a unique combination of postoperative units, staffing, expertise and technical equipment, which greatly influence postoperative triage decisions. Triage guidelines must therefore be adjusted to those characteristics, and developed by a local multidisciplinary team composed of surgeons, anaesthetists, pneumologists and intensivists.

In some institutions, all postsurgical patients are first transferred to the PACU before being transferred either to the ward or to the HDU. This offers more clinical information at the time of the final triage, since the first postoperative hours are a period of major physiological variation with the potential appearance of early pathological processes.

A transfer to the ward entails a substantial decrease in the quality and frequency of monitoring. The provision of a medical emergency team composed of anaesthetists and/or intensivists, may be a way to attenuate the risk [51] and may influence postoperative triage decisions.

\section{Prognosis of the lung cancer patient admitted to the ICU}

Series including mostly stage III-IV lung cancer have shown that the prognosis of lung cancer patient admitted to the ICU improved substantially with time. Before 2005, very high ICU mortality rates (46-85\%) were consistently reported; currently these have decreased to $13-47 \%$ [52]. Hospital mortality rates followed a similar decline from $75-91 \%$ to $24-65 \%$ [52]. However, we have to consider a possible selection bias, as arguments from pulmonologists and oncologists to admit patients to the ICU, as well as those advanced by intensivists to refuse them, could have changed during these periods in parallel to the introduction of new, more active anticancer treatments and improvement in intensive care management. 
Studies assessing prognostic factors are heterogeneous and of limited size. Among 13 studies, only seven included $>100$ patients [52]. The most frequently reported independent prognostic factors during ICU stay appeared to be performance status, acute respiratory failure, number of failing organs and need for vasopressors or mechanical ventilation. In addition, neoplastic airways obstruction, acute respiratory failure, cancer status, comorbidities, severity scores, sepsis and renal failure were predictive for in-hospital mortality. When long-term mortality was assessed, the cancer characteristics (presence of metastasis and cancer progression) retained their prognostic value.

RoQUEs et al. [53] showed, in 105 lung cancer patients requiring ICU stay, a 6-month mortality rate of $73 \%$; two-thirds of survivors received anti-cancer treatment. TOFFART et al. [54] showed that 3-month and 1-year survival rates were $37 \%$ and $12 \%$, respectively. A worsening of organ dysfunction at $72 \mathrm{~h}$ after ICU admission was associated with higher mortality (45\% versus $8 \%$ if organ dysfunction improved). 3-month survival was independent of noninvasive ventilation (NIV) requirement (40\% versus $47 \%$ without NIV), but was significantly decreased in patients requiring endotracheal intubation (22\%). For patients discharged alive from the ICU, the median survival after discharge was 104 days, the estimated survival being $54 \%$ at 90 days and $18 \%$ at 1 year.

The SEER (Surveillance, Epidemiology, and End Results) register analysed 49373 lung cancer patients admitted to the ICU for reasons other than surgical resection [55]. Of these, $76 \%$ survived the hospitalisation and 35\% were alive 6 months after discharge. Hospital mortality increased when mechanical ventilation was given, with 6-month survival only $15 \%$. A second study performed on the same database focused on 1134 patients aged $>65$ years with stage IIIB and IV nonsmall cell lung cancer (NSCLC) [56]. Mortality rates at 90 days and at 1 year were $71 \%$ and $90 \%$, respectively, with an in-hospital mortality rate of $33 \%$. Among patients discharged alive, $42 \%$ returned home while the others were transferred to rehabilitation services or palliative care institutions. Only $19 \%$ of patients received cancer treatment after hospital discharge.

\section{Specific complications and ICU management Surgical complications}

Even with optimal surgical and anaesthetic techniques, morbidity remains substantially elevated. Predominantly cardiac, pulmonary and pleural complications may arise in up to $83 \%$ of patients after extrapleural pneumonectomy with pericardial and diaphragmatic reconstruction [57]. Although minimally invasive procedures can be performed in high-risk patients with a lower complication rate, pre-existing clinical factors may be responsible for severe postoperative complications, justifying a close interaction between intensivists, anaesthetists, pneumologists, cardiologists, thoracic surgeons, medical oncologists and physiotherapists in the postoperative period.

\section{Cardiac arrhythmias}

Supraventricular tachyarrhythmias are more common than ventricular rhythm disturbances after noncardiac thoracic surgery [58]. Atrial fibrillation typically occurs between the second and fourth postoperative day in 10-20\% of patients after lobectomy, but in up to $40 \%$ after pneumonectomy [59]. Significant risk factors are right pneumonectomy, intrapericardial dissection, advanced age and reduced fluid intake. Adequate monitoring for several days is recommended and extensive guidelines are available from the American Association for Thoracic Surgery [60].

\section{Respiratory complications}

Pulmonary oedema is reported in up to $9 \%$ of patients after pneumonectomy and in $1-5 \%$ after lesser resection, with a high mortality rate $[61,62]$. It is a noncardiogenic, noninfectious pulmonary oedema that is considered to be a postresectional form of ARDS characterised by permeability oedema and diffuse alveolar damage. Therapy follows of the old adage "keep the patient dry"; reintubate or apply noninvasive ventilation in cases of respiratory failure along with the administration of inotropic/vasopressive drugs, while restricting fluid intake [63].

Postpneumonectomy syndrome is caused by extreme mediastinal shifting. Patients complain of progressive dyspnoea due to airway and vascular compression. In severe cases tissue expanders are used to reposition the mediastinum towards the midline [64].

Orthodeoxia-platypnoea syndrome is characterised by dyspnoea in a sitting position and is less pronounced when the patient lies down. It is related to reopening of a patent foramen ovale with an intracardiac shunt. A closing device can be used to obliterate the shunt [65].

Thromboembolic disease (either deep vein thrombosis or complicated by pulmonary embolism) is favoured by the hypercoagulability state induced by the cancer. Its incidence is up to $15 \%$ in lung cancer 
patients [66] and $1-5 \%$ in surgical patients [67]. Prevention by use of low molecular weight heparin, elastic stockings and early ambulation is of utmost importance. In high-risk patients the use of pneumatic compression devices is recommended. Management is not different from other thromboembolic diseases, except that it must be pursued for as long as the risk factor persists. Treatment modalities have switched over time to low molecular weight heparin [67, 68]; recently, in medical patients, direct oral anticoagulants have been demonstrated to be equally effective [69], but are not yet standard practice. For patients with severe haemodynamic compromise, thrombolytic therapy or pulmonary embolectomy may be applied.

Prolonged air leak is a common problem in patients with emphysema, which was reported in up to $50 \%$ of cases, rendering it the most frequent postoperative complication. Air leak is considered to be abnormal when it persists after 5 days [70]. Progressive subcutaneous and mediastinal emphysema may develop, eventually resulting in swollen face and eyes, the so-called "Michelin syndrome". Risk factors include severe bullous emphysema, lung volume reduction surgery and prolonged ventilatory support giving rise to barotrauma. Perioperative control of the bronchial stump and staple lines is recommended, if necessary by applying fibrin glue or other sealants, by reinforcement with strips or the creation of a pleural tent by detaching the parietal pleura. In a Cochrane systematic review, surgical sealants reduced postoperative air leaks and time to chest tube removal; however, a significant reduction in length of hospital stay could not be demonstrated [71]. Postoperatively, patients should be extubated as soon as possible to reduce the intrapulmonary pressure. Injection of talc, other sclerosing agents or autologous blood may be helpful to close a persisting parenchymal air leak. Insertion of a pigtail catheter with a Heimlich valve allows ambulatory treatment. For difficult cases, intrabronchial valves have been proposed to close alveolar pleural fistulas [72].

Lobar torsion is an uncommon $(<0.5 \%)$, but dramatic complication $[73,74]$. It occurs by the twisting of a remaining lobe around its bronchovascular pedicle, mainly, but not exclusively within the right middle lobe [75], with haemorrhagic infarction and lung necrosis. Diagnosis is obtained by bronchoscopy [76], and in most cases a completion pneumonectomy is needed. Prevention by fixation of the remaining lobe/s to surrounding tissue during the initial procedure is indicated [75].

\section{Specific pleural complications}

Persistent pleural space or a residual space, described as a "persisting pneumothorax" by thoracic radiologists, is observed after major lung resection less than after pneumonectomy. So-called "complicated residual spaces" are present when there is a persisting air leak or when colonisation with bacteria or fungi occur, giving rise to a pleural empyema. Drainage of the remaining pleural cavity is indicated [77]. Muscle flaps may be used to fill up the residual space in order to prevent recurrent infection. Pleural empyema is a rare event after lobectomy, but occurs in $2-12 \%$ of pneumectomies and is often associated with a bronchopleural fistula [73]. Risk factors include cardiovascular and pulmonary comorbidities, older age, malnutrition, neoadjuvant or induction therapy, diabetes, steroids, right pneumonectomy and extended resection, postoperative ARDS, pneumonia and prolonged mechanical ventilation. Pleural fluid culture before starting antibiotics is needed to detect the responsible bacteria and/or fungi, and antibacterial or antifungal treatment adapted as necessary. Initial therapy includes complete drainage of the pleural space in combination with antibiotics or antimycotics. Irrigation of the pleural space with an antiseptic solution may be necessary [78], and sometimes a temporary or definitive thoracostomy may be required to control a severe infection [79].

Bronchopleural fistula implies a direct communication between a remaining bronchial stump and the pleural space [80]. Clinical symptoms are quite similar to those of empyema and it is often heralded by a progressive inflammatory syndrome and respiratory distress. Massive expectoration of dark brown fluid may occur in the case of large fistula. Early cases are observed within 7 days of the procedure and are usually caused by inadequate or incomplete closure of the bronchial stump. Late cases are related to failure of healing of the bronchial stump, recurrent disease or progressive endobronchial or endotracheal infection. Risk factors are quite similar to empyema. Perioperative measures preventing bronchopleural fistula include covering of the bronchial stump with viable tissue [81]. Bronchoscopy is indicated to inspect the bronchial suture or staple line. Smaller fistulas may be detected by the injection of methylene blue at the stump. Management is similar to empyema and multidisciplinary collaboration is required for adequate treatment [82]. In cases of sudden expectoration of a large volume of fluid, the patient should be positioned with the head elevated and operated side down. Smaller fistulas may sometimes be obliterated bronchoscopically by the injection of glue or a sclerosing agent. Surgical closure of a larger bronchopleural fistula should be attempted after resolution of pleural infection, with excision of all necrotic tissue and covering of the remaining bronchial stump with viable tissue as a muscle flap, pericardium or omentum [73]. Postoperative irrigation of the thoracic cavity is indicated to prevent a recurrent empyema or fistulisation. 
Specific interventions of intensive care in thoracic oncology (other than surgical complications) Respiratory problems are the second most frequent cause of ICU admission in lung cancer patients [83]. The following events are commonly reported: febrile neutropenia and poststenotic infection (retro-obstructive pneumonia) (not addressed here), dyspnoea and acute respiratory failure, generally associated with pneumothorax or haemoptysis.

\section{Pneumothorax}

During the period of cancer staging, most ICU admissions are related to adverse events following tumour sampling. Transthoracic needle biopsies are complicated by pneumothoraxes in up to $60 \%$ of cases, but only a minority (up to 15\%) requires pleural drainage or admission to critical care [84]. Transbronchial biopsies have a lower incidence of complications [85]. Pneumothorax can appear a few hours after the procedure despite normal postprocedural radiography, which must be repeated. Pneumothorax may occur spontaneously due to tumour invasion or necrosis after chemotherapy. Severe respiratory failure is rare in the absence of other comorbidities, and pleural drainage is rapidly effective. Prolonged air leak is managed similarly to the postoperative setting.

\section{Intrabronchial bleeding}

Endoscopic biopsies rarely result in severe bleeding if standard precautions are taken: withholding anticoagulants and platelet antiaggregants, with the exception of low-dose aspirin. Massive haemoptysis can result from tumour infiltration or parenchymal necrosis following poststenotic infections, but rarely from thromboembolism. Haemoptysis from tumour growth is generally moderate, but massive bleeding due to the involvement of large vessels such as pulmonary arteries can occur. Admission to the ICU is recommended when bleeding is significant $(>200 \mathrm{~mL}$ per $24 \mathrm{~h}$ or $>50 \mathrm{~mL}$ per episode) or is responsible for respiratory distress (need for supplemental oxygen $>4-5 \mathrm{~L} \cdot \mathrm{min}^{-1}$ or arterial oxygen saturation measured by pulse oximetry $<88 \%$ ) or haemodynamic instability. Patients receiving anticoagulation and those who have extensive tissues necrosis but are potentially amenable to surgery should also be admitted. The diagnostic workup consists of a chest computed tomography scan and a bronchoscopy if the exact origin of the bleeding is unknown. Treatment modalities are oxygen administration and selective intubation in cases of massive bleeding. Bronchoscopy, preferably rigid bronchoscopy under general anaesthesia, can be used to coagulate intrabronchial lesions or to ensure proper selective intubation. Bronchial artery embolisation may sometimes be of limited value or have a transient effect. Surgery is sometimes feasible, but mortality is high. Antifibrinolytics are of limited, if any, use. In the absence of contraindications (mainly cardiac ischaemia and uncontrolled hypertension), vasopressin analogues show some effectiveness while waiting for interventional bronchoscopy, embolisation or surgery.

\section{Acute airway obstruction}

Acute obstruction of major airways arises in central tumours, due to intraluminal tumour growth, extraluminal compression or a mixed mechanism. With a few exceptions (severely functionally impaired patients), only tracheal or bilateral proximal obstructions generate significant respiratory distress. Dyspnoea is generally progressive, but can sometimes be acute, with stridor or wheezing depending on the level of obstruction. Ablation of the intraluminal tumoural component can be obtained by various techniques (laser, electrocoagulation or mechanical removal) if a visible lumen persists, followed by the implantation of endoluminal stents. Distal patent bronchi and normal lung are needed for insuring benefit. The procedure is performed under rigid bronchoscopy and general anaesthesia [86, 87].

Major pericardial and pleural fluid effusions

Intrathoracic malignancies regularly metastasize to pleura and/or pericardium, resulting in respiratory/ cardiac impairment [88]. With few exceptions, related to massive intracavitary bleeding the symptoms are progressive and are treated either by direct puncture (pleural/pericardial) or by drainage. Repeated puncture should be avoided since it poses a significant infectious risk and pleurodesis and/or pericardial drainage should be performed as soon as possible.

Some aetiologies of major dyspnoea

Rarely, dyspnoea can result from glottic oedema in the case of superior vena cava syndrome. The treatment relies on intravascular stenting and anticoagulation to prevent the extension of intracaval thrombosis. Corticosteroids and diuretics are ineffective [89]. Radiotherapy is not an emergency treatment, despite its potential effectiveness.

Progressive dyspnoea is frequently related to diffuse pulmonary involvement by lymphangitic or local spread. There is no specific therapy (including corticosteroids) showing activity outside of anticancer treatment, and ICU management relies only on administration of oxygen. Those patients should only be 
admitted to ICU if an active anticancer treatment can be provided [90]. Interstitial pneumonia may be related to side-effects of some tyrosine kinase inhibitors (TKIs). Sometimes, the cause of respiratory distress is difficult to pinpoint and more than one mechanism is involved. As these patients have frequent cardiac comorbidities, an $\mathrm{N}$-terminal pro-brain natriuretic peptide dosage can be ordered to rule out a cardiac aetiology [91].

\section{Advanced lung cancer and systemic anticancer treatment during ICU stay}

Prognosis of advanced lung cancer has changed over time with the introduction of new active chemotherapeutic agents, and for small subpopulations, very active targeted agents. Nevertheless, facing a persisting disease with poor prognosis, a few predictive factors for hospital mortality, including pre-existing poor performance status and weight loss, have to be considered before ICU admission [54, 92]. Other factors to be considered before ICU admission are the reversibility of the acute complication, such as desobstruction of proximal airways, therapeutic options for the lung cancer to be delivered after resolution of the acute complication, the absence of neoplastic progression [54] and the wishes of the patient. In a recent prospective study, TOFFART et al. [83] showed that the absence of refusal from the patients or their relatives is an independent factor for ICU referral.

Interest in administering anticancer treatment in ICU remains controversial, except for high-risk therapy (high-dose methotrexate, risk of severe allergic reaction, etc.). The level of evidence concerning the role of (urgent) chemotherapy in ICU is low, and most series deal with a mixed population, predominantly with haematological malignancies [93]. All studies demonstrated the feasibility of administering conventional chemotherapy, despite reduced prognosis for solid tumours [93, 94]. Prognostic factors reflect organ dysfunction (need for vasopressors, mechanical ventilation, hepatic failure and sequential organ failure assessment score [93-95].

Specific data for lung cancer are very limited. In a series of 20 small cell lung cancer (SCLC) patients, five received chemotherapy during their ICU stay [96]. Only two survived for $>200$ days. However, the introduction of targeted therapies with a rapid and very high response rate changed our pessimistic view, with case reports $[97,98]$ suggesting major activity of TKIs in patients with oncogenic driver mutations and respiratory failure, who were also on mechanical ventilation. The largest retrospective case-control study [90] included 14 patients with epidermal growth factor receptor mutation or anaplastic lymphoma kinase or ROS-1 rearrangements. Despite hospital mortality of 50\%, those patients with activating mutations receiving TKIs had reduced 30 -day mortality $(\mathrm{p}=0.07)$ and better survival (hazard ratio 0.12 , $\mathrm{p}=0.002$ ) than historical controls.

Due to its recent introduction into the armamentarium, there are no adequate data on immunotherapy and intensive care. However, by stimulating the immune system, these agents should probably be used with caution in patients presenting with modified immune systems (systemic inflammatory response syndrome, neutropenia, etc.).

Some indications for radiotherapy during ICU stay resulted from small series suggesting that irradiation for intubated patients could be beneficial. In a series of 26 patients (21 NSCLC, four SCLC), seven patients were extubated and six were discharged alive from the hospital [99].

Administering anticancer treatment in the ICU justifies an extensive discussion between the intensivist, the oncologist and patients or their relatives, taking into account the balance of benefit and risk. Specific populations with activating mutations should probably have more benefit in this setting with very active targeted therapies.

\section{Conclusion}

Patients with lung cancer will face intensive care management for two main reasons: postoperative setting or acute complications, related or not to their cancer or its treatment.

Careful preoperative assessment is mandatory to reduce postoperative morbidity and mortality. The anaesthetist plays a major role in decreasing postoperative risk and reducing the need for high-level postoperative monitoring. The trend for moving thoracic surgical postoperative care from the ICU towards HDUs or PACUs depends on the patient, procedure-related risk factors and on local specificities.

Severe cardiac and respiratory complications are the most common complications leading to ICU admission. Prevention and early recognition, as well as interdisciplinary cooperation are essential to obtain the best outcome [79]. Urgent anticancer treatment in patients suffering from respiratory failure due to tumoural infiltration yielded some success, mainly in cases of activating mutations/rearrangement, and should be discussed on a case-by-case basis. 
Although prognosis may be poor, admission to the ICU should be proposed to lung cancer patients after discussion between the patient and the multidisciplinary team, taking into account the possibility of reversal of the acute complication and the anticancer therapeutic options to be delivered during or after the ICU stay.

\section{References}

1 Allemani C, Coleman MP, CONCORD working group. Cancer survival: [corrected] the CONCORD-2 study authors' reply. Lancet 2015; 386: 429-430.

2 Caruso P, Ferreira AC, Laurienzo CE, et al. Short- and long-term survival of patients with metastatic solid cancer admitted to the intensive care unit: prognostic factors. Eur J Cancer Care 2010; 19: 260-266.

3 Soares M, Caruso P, Silva E, et al. Characteristics and outcomes of patients with cancer requiring admission to intensive care units: a prospective multicenter study. Crit Care Med 2010; 38: 9-15.

4 Berghmans T, Sculier JP. Is there any usefulness for a specific scoring system in assessing the prognosis of cancer patients admitted to the intensive care unit? Intensive Care Med 2004; 30: 1849.

5 Berghmans T, Paesmans M, Sculier JP. Is a specific oncological scoring system better at predicting the prognosis of cancer patients admitted for an acute medical complication in an intensive care unit than general gravity scores? Support Care Cancer 2004; 12: 234-239.

6 Sculier JP, Paesmans M, Markiewicz E, et al. Scoring systems in cancer patients admitted for an acute complication in a medical intensive care unit. Crit Care Med 2000; 28: 2786-2792.

7 Brunelli A, Charloux A, Bolliger CT, et al. ERS/ESTS clinical guidelines on fitness for radical therapy in lung cancer patients (surgery and chemo-radiotherapy). Eur Respir J 2009; 34: 17-41.

8 Diaper J, Ellenberger C, Villiger Y, et al. Comparison of cardiac output as assessed by transesophageal echo-Doppler and transpulmonary thermodilution in patients undergoing thoracic surgery. J Clin Anesth 2010; 22: 97-103.

9 Licker M, Diaper J, Villiger Y, et al. Impact of intraoperative lung-protective interventions in patients undergoing lung cancer surgery. Crit Care 2009; 13: R41.

10 Serpa Neto A, Hemmes SN, Barbas CS, et al. Incidence of mortality and morbidity related to postoperative lung injury in patients who have undergone abdominal or thoracic surgery: a systematic review and meta-analysis. Lancet Respir Med 2014; 2: 1007-1015.

11 Wolters U, Wolf T, Stützer $\mathrm{H}$, et al. ASA classification and perioperative variables as predictors of postoperative outcome. Br J Anaesth 1996; 77: 217-222.

12 Charlson ME, Pompei P, Ales KL, et al. A new method of classifying prognostic comorbidity in longitudinal studies: development and validation. J Chronic Dis 1987; 40: 373-383.

13 Birim O, Maat AP, Kappetein AP, et al. Validation of the Charlson comorbidity index in patients with operated primary non-small cell lung cancer. Eur J Cardiothorac Surg 2003; 23: 30-34.

14 Khuri SF, Daley J, Henderson W, et al. Risk adjustment of the postoperative mortality rate for the comparative assessment of the quality of surgical care: results of the National Veterans Affairs Surgical Risk Study. J Am Coll Surg 1997; 185: 315-327.

15 Glance LG, Lustik SJ, Hannan EL, et al. The Surgical Mortality Probability Model: derivation and validation of a simple risk prediction rule for noncardiac surgery. Ann Surg 2012; 255: 696-702.

16 Epstein SK, Faling LJ, Daly BD, et al. Predicting complications after pulmonary resection. Preoperative exercise testing $v s$ a multifactorial cardiopulmonary risk index. Chest 1993; 104: 694-700.

17 Ferguson MK, Durkin AE. A comparison of three scoring systems for predicting complications after major lung resection. Eur J Cardiothorac Surg 2003; 23: 35-42.

18 Falcoz PE, Conti M, Brouchet L, et al. The Thoracic Surgery Scoring System (Thoracoscore): risk model for in-hospital death in 15,183 patients requiring thoracic surgery. J Thorac Cardiovasc Surg 2007; 133: 325-332.

19 Lee TH, Marcantonio ER, Mangione CM, et al. Derivation and prospective validation of a simple index for prediction of cardiac risk of major noncardiac surgery. Circulation 1999; 100: 1043-1049.

20 Brunelli A, Varela G, Salati M, et al. Recalibration of the revised cardiac risk index in lung resection candidates. Ann Thorac Surg 2010; 90: 199-203.

21 Gupta PK, Gupta H, Sundaram A, et al. Development and validation of a risk calculator for prediction of cardiac risk after surgery. Circulation 2011; 124: 381-387.

22 Canet J, Gallart L, Gomar C, et al. Prediction of postoperative pulmonary complications in a population-based surgical cohort. Anesthesiology 2010; 113: 1338-1350.

23 Mazo V, Sabaté S, Canet J, et al. Prospective external validation of a predictive score for postoperative pulmonary complications. Anesthesiology 2014; 121: 219-231.

24 Berrisford R, Brunelli A, Rocco G, et al. The European Thoracic Surgery Database project: modelling the risk of in-hospital death following lung resection. Eur J Cardiothorac Surg 2005; 28: 306-311.

25 Brunelli A, Refai M, Xiumé F, et al. Performance at symptom-limited stair-climbing test is associated with increased cardiopulmonary complications, mortality, and costs after major lung resection. Ann Thorac Surg 2008; 86: $240-247$.

26 Brunelli A, Al Refai M, Monteverde M, et al. Predictors of early morbidity after major lung resection in patients with and without airflow limitation. Ann Thorac Surg 2002; 74: 999-1003.

27 Kozian A, Kretzschmar MA, Schilling T. Thoracic anesthesia in the elderly. Curr Opin Anaesthesiol 2015; 28: 2-9.

28 Licker M, Schnyder JM, Frey JG, et al. Impact of aerobic exercise capacity and procedure-related factors in lung cancer surgery. Eur Respir J 2011; 37: 1189-1198.

29 Bolliger CT, Wyser C, Roser H, et al. Lung scanning and exercise testing for the prediction of postoperative performance in lung resection candidates at increased risk for complications. Chest 1995; 108: 341-348.

30 Ebihara S, Saito H, Kanda A, et al. Impaired efficacy of cough in patients with Parkinson disease. Chest 2003; 124 1009-1015.

31 Dressler I, Fritzsche T, Cortina K, et al. Psychomotor dysfunction after remifentanil/propofol anaesthesia. Eur J Anaesthesiol 2007; 24: 347-354. 
32 Kim SW, Han HS, Jung HW, et al. Multidimensional frailty score for the prediction of postoperative mortality risk. JAMA Surg 2014; 149: 633-640.

33 Thomas PA, Berbis J, Baste JM, et al. Pneumonectomy for lung cancer: contemporary national early morbidity and mortality outcomes. J Thorac Cardiovasc Surg 2015; 149: 73-82.

34 Rosen JE, Hancock JG, Kim AW, et al. Predictors of mortality after surgical management of lung cancer in the National Cancer Database. Ann Thorac Surg 2014; 98: 1953-1960.

35 Lagarde SM, Reitsma JB, Maris AK, et al. Preoperative prediction of the occurrence and severity of complications after esophagectomy for cancer with use of a nomogram. Ann Thorac Surg 2008; 85: 1938-1945.

36 Melvan JN, Sancheti MS, Gillespie T, et al. Nonclinical factors associated with 30-day mortality after lung cancer: an analysis of 215,000 patients using the National Cancer Database. J Am Coll Surg 2015; 221: 550-563.

37 Grotenhuis BA, van Hagen P, Reitsma JB, et al. Validation of a nomogram predicting complications after esophagectomy for cancer. Ann Thorac Surg 2010; 90: 920-925.

38 Elshiekh MA, Lo TT Shipolini, AR, et al. Does muscle-sparing thoracotomy as opposed to posterolateral thoracotomy result in better recovery? Interact Cardiovasc Thorac Surg 2013; 16: 60-67.

39 Chowdhury MM, Dagash H, Pierro A. A systematic review of the impact of volume of surgery and specialization on patient outcome. Br J Surg 2007; 94: 145-161.

40 Ghaferi AA, Birkmeyer JD, Dimick JB. Variation in hospital mortality associated with inpatient surgery. $N$ Engl J Med 2009; 361: 1368-1375.

41 Ganter MT, Blumenthal S, Dübendorfer S, et al. The length of stay in the post-anaesthesia care unit correlates with pain intensity, nausea and vomiting on arrival. Perioper Med 2014; 3: 10.

42 Pöpping DM, Elia N, Marret E, et al. Protective effects of epidural analgesia on pulmonary complications after abdominal and thoracic surgery: a meta-analysis. Arch Surg 2008; 143: 990-999.

43 Powell ES, Cook D, Pearce AC, et al. A prospective, multicentre, observational cohort study of analgesia and outcome after pneumonectomy. Br J Anaesth 2011; 106: 364-370.

44 Gawande AA, Kwaan MR, Regenbogen SE, et al. An Apgar score for surgery. J Am Coll Surg 2007; 204: 201-208.

45 Haynes $\mathrm{AB}$, Regenbogen SE, Weiser TG, et al. Surgical outcome measurement for a global patient population: validation of the Surgical Apgar Score in 8 countries. Surgery 2011; 149: 519-524.

46 Brunelli A, Fianchini A, Gesuita R, et al. POSSUM scoring system as an instrument of audit in lung resection surgery. Physiological and operative severity score for the enumeration of mortality and morbidity. Ann Thorac Surg 1999; 67: 329-331.

47 Prytherch DR, Whiteley MS, Higgins B, et al. POSSUM and Portsmouth POSSUM for predicting mortality. Physiological and Operative Severity Score for the enUmeration of Mortality and morbidity. Br J Surg 1998; 85: $1217-1220$.

48 Nasraway SA, Cohen IL, Dennis RC, et al. Guidelines on admission and discharge for adult intermediate care units. American College of Critical Care Medicine of the Society of Critical Care Medicine. Crit Care Med 1998; 26: 607-610.

49 Guidelines for intensive care unit admission, discharge, and triage. Task Force of the American College of Critical Care Medicine, Society of Critical Care Medicine. Crit Care Med 1999; 27: 633-638.

50 Schweizer A, Khatchatourian G, Höhn L, et al. Opening of a new postanesthesia care unit: impact on critical care utilization and complications following major vascular and thoracic surgery. J Clin Anesth 2002; 14: 486-493.

51 Bellomo R, Goldsmith D, Uchino S, et al. Prospective controlled trial of effect of medical emergency team on postoperative morbidity and mortality rates. Crit Care Med 2004; 32: 916-921.

52 Meert AP, Berghmans T, Sculier JP. Le patient atteint de cancer bronchique en reanimation. [The patient with lung cancer in intensive care]. Rev Mal Respir 2014; 31: 961-974.

53 Roques S, Parrot A, Lavole A, et al. Six-month prognosis of patients with lung cancer admitted to the intensive care unit. Intensive Care Med 2009; 35: 2044-2050.

54 Toffart AC, Minet C, Raynard B, et al. Use of intensive care in patients with nonresectable lung cancer. Chest 2011; 139: 101-108.

55 Cooke CR, Feemster LC, Wiener RS, et al. Aggressiveness of intensive care use among patients with lung cancer in the Surveillance, Epidemiology, and End Results - Medicare registry. Chest 2014; 146: 916-923.

56 Slatore CG, Cecere LM, Letourneau JL, et al. Intensive care unit outcomes among patients with lung cancer in the Surveillance, Epidemiology, and End Results - Medicare registry. J Clin Oncol 2012; 30: 1686-1691.

57 Van Schil PE, Baas P, Gaafar R, et al. Trimodality therapy for malignant pleural mesothelioma: results from an EORTC phase II multicentre trial. Eur Respir J 2010; 36: 1362-1369.

58 Ferguson MK, Saha-Chaudhuri P, Mitchell JD, et al. Prediction of major cardiovascular events after lung resection using a modified scoring system. Ann Thorac Surg 2014; 97: 1135-1140.

59 De Decker K, Jorens PG, Van Schil P. Cardiac complications after noncardiac thoracic surgery: an evidence-based current review. Ann Thorac Surg 2003; 75: 1340-1348.

60 Frendl G, Sodickson AC, Chung MK, et al. 2014 AATS guidelines for the prevention and management of perioperative atrial fibrillation and flutter for thoracic surgical procedures. Executive summary. $J$ Thorac Cardiovasc Surg 2014; 148: 772-791.

61 Waller DA, Keavey P, Woodfine L, et al. Pulmonary endothelial permeability changes after major lung resection. Ann Thorac Surg 1996; 61: 1435-1440.

62 Licker M, Fauconnet P, Villiger Y, et al. Acute lung injury and outcomes after thoracic surgery. Curr Opin Anaesthesiol 2009; 22: 61-67.

63 Parquin F, Marchal M, Mehiri S, et al. Post-pneumonectomy pulmonary edema: analysis and risk factors. Eur J Cardiothorac Surg 1996; 10: 929-932.

64 Villeneuve PJ, Sundaresan S. Complications of pulmonary resection: postpneumonectomy pulmonary edema and postpneumonectomy syndrome. Thorac Surg Clin 2006; 16: 223-234.

65 Delalieux S, De Greef K, Hendriks J, et al. Orthodeoxia-platypnea syndrome presenting as paradoxical peripheral embolism. Ann Thorac Surg 2008; 85: 1798-1800.

66 Crolow C, Samulowski M, Blum T, et al. Häufigkeit thromboembolischer Komplikationen bei Patienten mit Lungenkarzinom. [Frequency of thromboembolic complications in patients with lung cancer]. Pneumologie 2013; 67: 442-447. 
67 Brunelli A. Deep vein thrombosis/pulmonary embolism: prophylaxis, diagnosis, and management. Thorac Surg Clin 2012; 22: 25-28.

68 Lyman GH, Khorana AA, Kuderer NM, et al. Venous thromboembolism prophylaxis and treatment in patients with cancer: American Society of Clinical Oncology clinical practice guideline update. J Clin Oncol 2013; 31: 2189-2204.

69 Vedovati MC, Germini F, Agnelli G, et al. Direct oral anticoagulants in patients with VTE and cancer: a systematic review and meta-analysis. Chest 2015; 147: 475-483.

70 Mueller MR, Marzluf BA. The anticipation and management of air leaks and residual spaces post lung resection. J Thorac Dis 2014; 6: 271-284.

71 Belda-Sanchís J, Serra-Mitjans M, Iglesias Sentis M, et al. Surgical sealant for preventing air leaks after pulmonary resections in patients with lung cancer. Cochrane Database Syst Rev 2010; 1: CD003051.

72 Mahajan AK, Doeing DC, Hogarth DK. Isolation of persistent air leaks and placement of intrabronchial valves. J Thorac Cardiovasc Surg 2013; 145: 626-630.

73 Crabtree TD, Denlinger CE. Complications of surgery for lung cancer. In: Pass HI, Carbone D, Johnson DH, et al., eds. Principles and Practice of Lung Cancer. Philadelphia, Wolters Kluwer Health/Lippincott Williams \& Wilkins, 2010; pp. 531-546.

74 Cable DG, Deschamps C, Allen MS, et al. Lobar torsion after pulmonary resection: presentation and outcome. J Thorac Cardiovasc Surg 2001; 122: 1091-1093.

75 Hendriks J, Van Schil P, De Backer W, et al. Massive cerebral infarction after completion pneumonectomy for pulmonary torsion. Thorax 1994; 49: 1274-1275.

76 Alassar A, Marchbank A. Left lower lobe torsion following upper lobectomy - prompt recognition and treatment improve survival. J Surg Case Rep 2014; 2014: rju078.

77 Murthy SC. Air leak and pleural space management. Thorac Surg Clin 2006; 16: 261-265.

78 Gharagozloo F, Margolis M, Facktor M, et al. Postpneumonectomy and postlobectomy empyema. Thorac Surg Clin 2006; 16: 215-222.

79 Van Schil PE, Hendriks JM, Lauwers P. Focus on treatment complications and optimal management surgery. Transl Lung Cancer Res 2014; 3: 181-186.

80 Asamura H, Naruke T, Tsuchiya R, et al. Bronchopleural fistulas associated with lung cancer operations. Univariate and multivariate analysis of risk factors, management, and outcome. J Thorac Cardiovasc Surg 1992; 104: $1456-1464$.

81 Llewellyn-Bennett R, Wotton R, West D. Prophylactic flap coverage and the incidence of bronchopleural fistulae after pneumonectomy. Interact Cardiovasc Thorac Surg 2013; 16: 681-685.

82 Leuzzi G, Facciolo F, Pastorino U, et al. Methods for the postoperative management of the thoracic oncology patients: lessons from the clinic. Expert Rev Respir Med 2015; 9: 751-767.

83 Toffart AC, Pizarro CA, Schwebel C, et al. Selection criteria for intensive care unit referral of lung cancer patients: a pilot study. Eur Respir J 2015; 45: 491-500.

84 Manhire A, Charig M, Clelland C, et al. Guidelines for radiologically guided lung biopsy. Thorax 2003; 58 : 920-936.

85 Wang Memoli JS, Nietert PJ, Silvestri GA. Meta-analysis of guided bronchoscopy for the evaluation of the pulmonary nodule. Chest 2012; 142: 385-393.

86 Bolliger CT, Mathur PN, Beamis JF, et al. ERS/ATS statement on interventional pulmonology. European Respiratory Society/American Thoracic Society. Eur Respir J 2002; 19: 356-373.

87 Bolliger CT, Sutedja TG, Strausz J, et al. Therapeutic bronchoscopy with immediate effect: laser, electrocautery, argon plasma coagulation and stents. Eur Respir J 2006; 27: 1258-1271.

88 Antony VB, Loddenkemper R, Astoul P, et al. Management of malignant pleural effusions. Eur Respir J 2001; 18 : 402-419.

89 Wilson LD, Detterbeck FC, Yahalom J. Clinical practice. Superior vena cava syndrome with malignant causes. N Engl J Med 2007; 356: 1862-1869.

90 Toffart AC, Dhalluin X, Girard N, et al. Patients with advanced lung cancer harboring oncogenic mutations should be admitted to intensive care units. Intensive Care Med 2015; 41: 164-165.

91 Maisel AS, Krishnaswamy P, Nowak RM, et al. Rapid measurement of B-type natriuretic peptide in the emergency diagnosis of heart failure. N Engl J Med 2002; 347: 161-167.

92 Christodoulou C, Rizos M, Galani E, et al. Performance status (PS): a simple predictor of short-term outcome of cancer patients with solid tumors admitted to the intensive care unit (ICU). Anticancer Res 2007; 27: 2945-2948.

93 Song JU, Suh GY, Chung MP, et al. Risk factors to predict outcome in critically ill cancer patients receiving chemotherapy in the intensive care unit. Support Care Cancer 2011; 19: 491-495.

94 Darmon M, Thiery G, Ciroldi M, et al. Intensive care in patients with newly diagnosed malignancies and a need for cancer chemotherapy. Crit Care Med 2005; 33: 2488-2493.

95 Benoit DD, Depuydt PO, Vandewoude KH, et al. Outcome in severely ill patients with hematological malignancies who received intravenous chemotherapy in the intensive care unit. Intensive Care Med 2006; 32: 93-99.

96 Jennens RR, Rosenthal MA, Mitchell P, et al. Outcome of patients admitted to the intensive care unit with newly diagnosed small cell lung cancer. Lung Cancer 2002; 38: 291-296.

97 Bosch-Barrera J, Sais E, Lorencio C, et al. Successful empirical erlotinib treatment of a mechanically ventilated patient newly diagnosed with metastatic lung adenocarcinoma. Lung Cancer 2014; 86: 102-104.

98 Ahn HK, Jeon $\mathrm{K}$, Yoo $\mathrm{H}$, et al. Successful treatment with crizotinib in mechanically ventilated patients with ALK positive non-small-cell lung cancer. J Thorac Oncol 2013; 8: 250-253.

99 Louie AV, Lane S, Palma DA, et al. Radiotherapy for intubated patients with malignant airway obstruction: futile or facilitating extubation? J Thorac Oncol 2013; 8: 1365-1370. 